\title{
A potential reduction interior point method for semi-infinite linear programming on a Hilbert space
}

\author{
Andrew E.B. Lim \& John B. Moore \\ Department of Systems Engineering \\ Research School of Information Sciences and Engineering \\ The Australian National University \\ Canberra, ACT 0200 AUSTRALIA \\ Fax : + 6162798688 e-mail: andrew@ syseng.anu.edu.au
}

Keywords: Optimization; interior point algorithms; semiinfinite linear programming; potential reduction algorithm.

\begin{abstract}
We consider a semi-infinite linear programming problem where the variables may belong to an infinite-dimensional Hilbert space. We generalize a potential reduction method introduced by Ye so that it can be used to solve this problem. Furthermore, convergence of the iterates produced by this algorithm to the optimal solution is proven. As an example, we show how this algorithm can be used to solve continuous linear programming (CLP) problems.
\end{abstract}

\section{Introduction}

Many optimization problems are naturally cast as semiinfinite problems; for instance, continuous time, optimal control problems subject to all time state constraints. In this paper, we consider a semi-infinite linear programming problem where the variables may belong to an infinite-dimensional Hilbert space. We generalize a potential reduction method introduced by Ye [9] so that it can be used to solve this problem.

Although much of the research on interior point methods (IPM's) has focused on finite-dimensional problems, interest in infinite-dimensional problems has begun to attract more attention, especially of late. In recent papers by Faybusovich and Moore [1, 2] the logarithmic barrier method is extended so as to be amendable to infinite-dimensional quadratic optimization problems subject to finitely many linear or quadratic constraints. On the other hand, Ferris and Philpott [3, 4], Powell [6] and Todd [8] study a class of semi-infinite linear programming problems. Todd considers a family of semiinfinite LP problems on the space of continuous functionals and shows that a concept which he calls invariance is fundamental to whether a given IPM can be generalized to solve the limiting semi-infinite problem on $C[0,1]$. He determines which IPM's converge to a sensible limiting algorithm as the number of constraints tends to infinity. Consequently, he does not deal with the issue of convergence of the iterates produced by the limiting algorithm. In particular, he shows that a potential reduction method introduced by Ye [9] has a sensible generalization to the semi-infinite case on $C[0,1]$. In the papers by Ferris and Philpott [3, 4] and Powell [6] the affine scaling algorithm, and Karmarkar's algorithm respectively are generalized to the semi-infinite setting.

In this paper we combine features of $[1,2]$ and $[3,4,6,8]$ by studying semi-infinite LP where the variable can belong to an arbitrary infinite-dimensional Hilbert space (as opposed to a space of continuous functionals). We generalize a potential reduction method introduced by Ye [9]. Furthermore, convergence of the iterates produced by this algorithm to the optimal solution is proven. As an example, we show how this algorithm can be used to solve continuous linear programming (CLP) problems [7].

\section{Semi-infinite LP and the Potential Function.}

Let $(H,\langle\cdot, \cdot\rangle)$ be a Hilbert Space and $X$ a closed subspace of $H$. For $i=1, \cdots, m$ and $\eta \in[0,1]$ let $a_{i}(\eta) \in H, b_{i}(\eta) \in \mathbf{R}$ and $c \in X$ be given. Assume that $a_{i}(\eta)$ and $b_{i}(\eta)$ depend continuously on $\eta \in[0,1]$. Furthermore, assume that

$$
X=\operatorname{span}\left\{a_{i}(\eta): i=1, \ldots, m \text { and } \eta \in[0,1]\right\}
$$

We consider the semi-infinite linear programming problem which has a primal form

$$
(P)\left\{\begin{array}{c}
\langle c, x\rangle \rightarrow \min \\
\left\langle a_{i}(\eta), x\right\rangle \leq b_{i}(\eta), \quad i=1, \cdots, m, \eta \in[0,1] \\
x \in X
\end{array}\right.
$$


The problem dual to $(P)$ is

$$
(D)\left\{\begin{array}{c}
-\sum_{i=1}^{m} \int_{0}^{1} b_{i}(\eta) \dot{v}_{i}(\eta) d \eta \rightarrow \max \\
c+\sum_{i=1}^{m} \int_{0}^{1} a_{i}(\eta) \dot{v}_{i}(\eta) d \eta=0 \\
v_{i}(\eta) \in B V[0,1] \\
\dot{v}_{i}(\eta) \geq 0
\end{array}\right.
$$

We make the following assumptions:

Assumption 2.1 There exists an optimal solution $x^{*} \in X$ for (P) such that $\left\langle c, x^{*}\right\rangle>-\infty$

Assumption 2.2 There exists $x \in X$ such that $\left\langle a_{i}(\eta), x\right\rangle<$ $b_{i}(\eta)$ for $i=1, \cdots, m$ and $\eta \in[0,1]$.

Assumption 2.3 There is $0<M<\infty$ such that $\|x\| \leq M$ for every primal feasible $x \in X$.

It is well known that if $v_{i}(\eta) \in B V[0,1]$ is dual feasible (ie. feasible for $(D))$ then

$$
-\sum_{i=1}^{m} \int_{0}^{1} b_{i}(\eta) \dot{v}_{i}(\eta) d \eta \leq z^{*} \leq\langle c, x\rangle
$$

where $z^{*}$ is the optimal cost for $(P)$ and $(D)$. Let $z \leq z^{*}$. Suppose that $x \in H$ is primal feasible. The primal potential function for $(P)$ is

$$
\phi(x, z)=\rho \ln [\langle c, x\rangle-z]-\sum_{i=1}^{m} \int_{0}^{1} \ln s_{i}(\eta, x) d \eta
$$

where $s_{i}(\eta)=b_{i}(\eta)-\left\langle a_{i}(\eta), x\right\rangle$. For every primal feasible $x^{0} \in X$, it follows from Assumption 2.3 that there exists $0<K\left(x^{0}\right)<\infty$ such that

$$
K\left(x^{0}\right)>\exp \left[\frac{\int_{0}^{1} s_{i}(x, \eta) d \eta-\int_{0}^{1} s_{i}\left(x^{0}, \eta\right) d \eta}{\rho}\right]
$$

for every primal feasible $x \in X$. Moreover

Proposition 2.1 Let $\epsilon>0$ be given. If $x, x^{0} \in X$ are primal feasible and $z, z^{0}$ are lower bounds of $z^{*}$, then

$$
\phi(x, z)<\phi\left(x^{0}, z^{0}\right)+\rho \ln \frac{\epsilon}{K\left(x^{0}\right) \cdot\left(\left\langle c, x^{0}\right\rangle-z^{0}\right)}
$$

implies that $\langle c, x\rangle-z<\epsilon$.

Proof: The interested reader should consult [5].

Therefore, by making $\phi(x, z)$ sufficiently negative, the duality gap can be made sufficiently small.

\section{Algorithm}

We now develop an interior point algorithm which can be used to reduce $\phi(x, z)$.
Let $X(\eta)=\left[X_{1}(\eta), \cdots, X_{m}(\eta)\right]^{\prime}$ where $X_{j}(\eta) \in C[0,1]$. Define $\|\cdot\|_{2}$ and $\|\cdot\|_{\infty}$ as follows:

$$
\begin{aligned}
\|X(\eta)\|_{2} & =\left[\sum_{i=1}^{m} \int_{0}^{1}\left|X_{i}(\eta)\right|^{2} d \eta\right]^{\frac{1}{2}} \\
\|X(\eta)\|_{\infty} & =\sup _{1 \leq i \leq m} \sup _{\eta \in[0,1]}\left|X_{i}(\eta)\right|
\end{aligned}
$$

Given primal feasible solutions $x^{0}, x^{1} \in H$, let

$$
\left\langle\frac{a(\eta)}{s\left(\eta, x^{0}\right)}, x^{1}-x_{0}\right\rangle=\left[\begin{array}{c}
\left\langle\frac{a_{1}(\eta)}{s_{1}\left(\eta, x^{0}\right)}, x^{1}-x^{0}\right\rangle \\
\vdots \\
\left\langle\frac{a_{m}(\eta)}{s_{1}\left(\eta, x^{0}\right)}, x^{1}-x^{0}\right\rangle
\end{array}\right]
$$

Note that

$$
\left\langle\frac{a_{j}(\eta)}{s_{j}\left(\eta, x^{0}\right)}, x^{1}-x^{0}\right\rangle \in C[0,1]
$$

The following is an infinite dimensional generalization of a result from [9], a proof of which appears in [5].

Proposition 3.1 Let $x^{0}, x^{1} \in X$ be primal feasible and $z^{0} \leq$ $z^{*}$ where $z^{*}$ satisfies (1). If

$$
\left\|\left\langle\frac{a(\eta)}{s\left(\eta, x^{0}\right)}, x^{1}-x^{0}\right\rangle\right\|_{\infty}<1
$$

then

$$
\begin{array}{r}
\phi\left(x^{1}, z^{0}\right)-\phi\left(x^{0}, z^{0}\right) \leq\left\langle\nabla \phi\left(x^{0}, z^{0}\right), x^{1}-x^{0}\right\rangle \\
+\frac{\left\|\left\langle\frac{a(\eta)}{s\left(\eta, x^{0}\right)}, x^{1}-x^{0}\right\rangle\right\|_{2}^{2}}{2\left\{1-\left\|\left\langle\frac{a(\eta)}{s\left(\eta, x^{0}\right)}, x^{1}-x^{0}\right\rangle\right\|_{\infty}\right\}}
\end{array}
$$

where

$$
\nabla \phi(x, z)=\frac{\rho}{\langle c, x\rangle-z} c+\sum_{i=1}^{m} \int_{0}^{1} \frac{a_{i}(\eta)}{s_{i}(\eta, x)} d \eta
$$

Given a primal feasible solution $x^{0} \in X$, we can use (4) to determine another primal feasible solution $x^{1} \in X$ which minimizes the upper bound on the difference

$$
\phi\left(x^{1}, z^{0} x\right)-\phi\left(x^{0}, z^{0}\right)
$$

in the following way: we choose a primal feasible $x^{1} \in H$ which minimizes the right hand side of (4). Noting that

$$
\left\|\left\langle\frac{a(\eta)}{s\left(\eta, x^{0}\right)}, x^{1}-x^{0}\right\rangle\right\|_{2}^{2}=\left\langle Q\left(x^{0}\right) \cdot\left(x^{1}-x^{0}\right), x^{1}-x^{0}\right\rangle
$$

where

$$
Q\left(x^{0}\right)=\sum_{i=1}^{m} \int_{0}^{1} \frac{a_{i}(\eta) \otimes a_{i}(\eta)}{s_{i}\left(\eta, x^{0}\right)^{2}} d \eta
$$


we obtain from (4) the following problem over $x$ :

$$
\begin{gathered}
\left\langle\nabla \phi\left(x^{0}, z^{0}\right), x-x^{0}\right\rangle \rightarrow \min \\
\left\langle Q\left(x^{0}\right) \cdot\left(x-x^{0}\right), x-x^{0}\right\rangle^{\frac{1}{2}} \leq \beta^{*}<1 \\
x-x^{0} \in X
\end{gathered}
$$

Note that the inequality (4) holds only if (3) is satisfied. On the other hand, unless $\beta=0$ the inequality in (7) being true will not guarantee that (3) is true either. That is, a solution $x^{1}$ of (7), for $0<\beta^{*}<1$ will generally not give rise to a minimal upper bound on the difference (5) as suggested by the inequality (4). For the moment, we assume that there exists $0<\beta^{*}<1$ such that the optimal solution of (7) gives rise to a solution $x^{1}$ which satisfies the inequality (3) and hence (4). We defer the issue of calculating $\beta^{*}$ until later and for the moment, assume that $\beta^{*}$ has been found. Since $Q\left(x^{0}\right): X \rightarrow X$ is a strictly positive operator, on $X$ for each $x^{0} \in X$, it follows that $\langle\cdot, \cdot\rangle_{x^{0}}: X \times X \rightarrow \mathbf{R},\langle\cdot, \cdot\rangle_{x^{0}}=\left\langle Q\left(x^{0}\right) \cdot, \cdot\right\rangle$ defines an inner product on $X$ and hence, a Riemannian metric on $X$. The gradient of $\phi(x, z)$ with respect to this Riemannian metric is given as follows.

Lemma 3.1 Let $Q\left(x^{0}\right): X \rightarrow X$ be given by (6). The gradient of $\phi\left(x^{0}, z^{0}\right)$ with respect to the Riemannian metric defined by the inner product $\langle\cdot, \cdot\rangle_{s}$ is

$$
\nabla_{x^{0}} \phi\left(x^{0}, z^{0}\right)=Q\left(x^{0}\right)^{-1} \nabla \phi\left(x^{0}, z^{0}\right)
$$

Proof: The functional $\left\langle\nabla \phi\left(x^{0}, z^{0}\right), \cdot\right\rangle: X \rightarrow \mathbf{R}$ is a bounded linear functional on the Hilbert (sub)space $X$, so by the Riesz theorem for functionals on a Hilbert space, there exists a unique $\nabla_{x^{\circ}} \phi\left(x^{0}, z^{0}\right) \in X$ such that

$$
\left\langle\nabla \phi\left(x^{0}, z^{0}\right), y\right\rangle=\left\langle Q\left(x^{0}\right) \cdot \nabla_{x^{0}} \phi\left(x^{0}, z^{0}\right), y\right\rangle
$$

for all $y \in X$. Therefore

$$
\nabla \phi\left(x^{0}, z^{0}\right)-Q\left(x^{0}\right) \cdot \nabla_{x^{0}} \phi\left(x^{0}, z^{0}\right) \in X^{\perp}
$$

The result follows from the fact that

$$
\nabla \phi\left(x^{0}, z^{0}\right)-Q\left(x^{0}\right) \cdot \nabla_{x^{0}} \phi\left(x^{0}, z^{0}\right) \in X
$$

From Proposition 2.1, it follows that the optimal solution $x^{1}$ of (7) is

$$
x^{1}=x^{0}-\beta^{*} \frac{Q\left(x^{0}\right)^{-1} \cdot \nabla \phi\left(x^{0}, z^{0}\right)}{\left\langle Q\left(x^{0}\right)^{-1} \cdot \nabla \phi\left(x^{0}, z^{0}\right), \nabla \phi\left(x^{0}, z^{0}\right)\right\rangle^{\frac{1}{2}}}
$$

We return now to the question of choosing $\beta^{*}$. Recall that in general, $0<\beta^{*}<1$ will not guarantee that (3) holds and hence, will not guarantee the reduction in $\phi(x, z)$ which (4) suggests. However, if $\beta^{*}$ is calculated as follows, a reduction in the potential function is guaranteed. The following is an infinite dimensional generalization of a result from [9] and the interested reader should consult [5] for a derivation. Let $\pi \in X$ be given by

$$
\pi=\frac{Q\left(x^{0}\right)^{-1} \cdot \nabla \phi\left(x^{0}, z^{0}\right)}{\left\langle Q\left(x^{0}\right)^{-1} \cdot \nabla \phi\left(x^{0}, z^{0}\right), \nabla \phi\left(x^{0}, z^{0}\right)\right\rangle^{\frac{1}{2}}}
$$

Suppose that $0<\beta^{*} \leq \frac{1}{2}$ satisfies the inequality

$$
\beta^{*}\left|\left\langle\frac{a_{i}(\eta)}{s_{i}\left(\eta, x^{0}\right)}, \pi\right\rangle\right|<1 \quad i=\frac{1}{2}, \cdots, m ; \eta \in[0,1]
$$

It can then be shown that

$$
\phi\left(x^{0}-\beta^{*} \pi, z^{0}\right)-\phi\left(x^{0}, z^{0}\right) \leq-\frac{\beta^{*}}{2}\left\langle Q\left(x^{0}\right) \cdot \pi, \pi\right\rangle=-\frac{\beta^{*}}{2}
$$

Unlike the finite dimensional case, a reduction of $\phi\left(x^{0}, z\right)$ by at least a fixed constant after each iteration is not guaranteed because $\beta^{*}$ depends on $x^{0}$. Thus, (11) on its own does not guarantee that $\phi(x, z)$ can be made arbitrarily negative. Also, for this reason the theoretically attractive upper bound polynomial complexity on the number of iterates does not hold.

We now consider the issue of updating a lower bound $z^{0}$ of $z^{*}$. Let $x^{0} \in X$ be a primal feasible solution. It can be shown that $v(\eta) \in B V[0,1]$ satisfying

$$
\dot{v}_{i}(\eta)=f\left(x^{0}, z^{0}, \eta\right)
$$

where

$$
\begin{aligned}
f_{i}\left(x^{0}, z^{0}, \eta\right)= & {\left[\frac{\left\langle c, x^{0}\right\rangle-z^{0}}{\rho}\right]\left[s_{i}\left(\eta, x^{0}\right)^{-1}\right.} \\
& \left.\left\langle\frac{a_{i}(\eta)}{s_{i}\left(\eta, x^{0}\right)^{2}}, Q\left(x^{0}\right)^{-1} \cdot \nabla \phi\left(x^{0}, z^{0}\right)\right\rangle\right]
\end{aligned}
$$

is dual feasible. The lower bound $z^{0}$ can be increased to $z^{1}>$ $z^{0}$ so long as

$$
f_{i}\left(x^{0}, z^{1}, \eta\right) \geq 0, \quad i=1, \cdots, m ; \eta \in[0,1]
$$

Hence, $z^{1}$ can be obtained by solving:

$$
(Z P)\left\{\begin{array}{c}
z^{1}=\arg \max z \\
f_{i}\left(x^{0}, z, \eta\right) \geq 0 ; \quad i=1, \cdots, m ; \eta \in[0,1] \\
z \geq z^{0}
\end{array}\right.
$$

If $z^{1}$ can be made arbitrarily large, then $(P)$ has no solution. Note also that for $\infty>z^{1}>z^{0}$

$$
\phi\left(x^{0}, z^{1}\right)<\phi\left(x^{0}, z^{0}\right)
$$

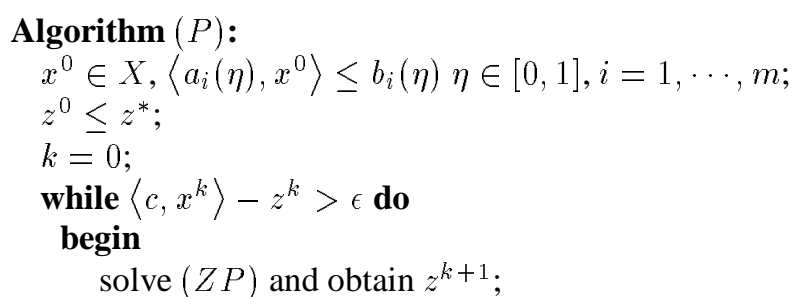




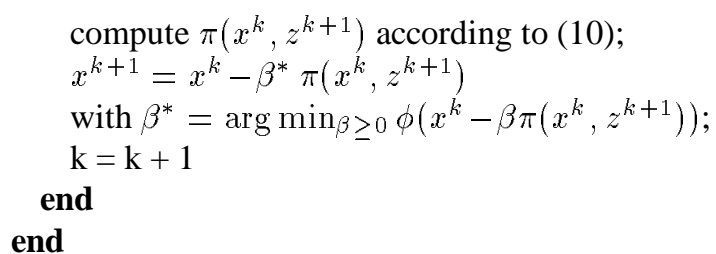

\section{Convergence results}

We consider now the convergence of the iterates $\left(x^{k}, z^{k}\right)$ produced by Algorithm $(P)$. Consider the following discretization of $(P)$ : let $I_{N} \subseteq[0,1]$ be a finite subset of the form

$$
I_{N}=\left\{\frac{i}{2^{N}}: i=1, \cdots, 2^{N}\right\}
$$

which partitions $[0,1]$ into $2^{N}$ subintervals. Clearly $I_{N} \subset I_{N+1}$. Let $c_{N} \in X_{N}$ be the orthogonal projection of $c \in X$ onto the subspace

$$
X_{N}=\operatorname{span}\left\{a_{i}(\eta): i=1, \cdots, m ; \eta \in I_{N}\right\}
$$

A finite-dimensional discretization of $(P)$ with respect to the partition $I_{N}$ is:

$$
\left(P_{N}\right)\left\{\begin{array}{c}
\left\langle c_{N}, x\right\rangle \rightarrow \min \\
\left\langle a_{i}(\eta), x\right\rangle \leq b_{i}(\eta) ; \quad \eta \in I_{N}, i=1, \cdots, m \\
x \in X
\end{array}\right.
$$

Dual to $\left(P_{N}\right)$ is

$$
\left(D_{N}\right)\left\{\begin{array}{c}
-\frac{1}{2^{N}} \sum_{i=1}^{m} \sum_{i=1}^{2^{N}} \lambda_{i j} b_{i}\left(\eta_{j}\right) \rightarrow \max \\
c_{N}+\frac{1}{2^{N}} \sum_{i=1}^{m} \sum_{i=1}^{2^{N}} \lambda_{i j} a_{i}\left(\eta_{j}\right)=0 \\
\lambda_{i j} \geq 0
\end{array}\right.
$$

where $\eta_{i}=\frac{i}{2^{N}}$. Assuming that an optimal solution of $\left(P_{N}\right)$ exists, it follows from $X=X_{N} \oplus\left(X_{N}^{\perp} \cap X\right)$ and $a_{i}\left(\eta_{j}\right), c_{N} \in X_{N}$, that there is $x_{N}^{*} \in X_{N}$ which achieves this optimal cost. Since $X_{N}$ is a finite dimensional subset of $X$, it follows that $\left(P_{N}\right)$ is equivalent to a finite-dimensional LP problem over $\mathbf{R}^{q(N)}$ where $q(N)=m \cdot 2^{N}$. Let $x_{N}^{*}$ denote the optimal solution for $\left(P_{N}\right)$. Then the sequence $\left\{x_{N}^{*}\right\}_{N=1}^{\infty}$ has the following convergence properties.

Lemma 4.1 Let $x_{N}^{*}$ denote the optimal solution for the problem $\left(P_{N}\right)$ and $x^{*}$ the optimal solution for $(P)$. Then $\left\langle c_{N}, x_{N}^{*}\right\rangle \rightarrow\left\langle c, x^{*}\right\rangle$.

Proposition 4.1 If $x^{*}$ is the optimal solution for $(P)$ then $x_{N}^{*} \rightarrow x^{*}$ as $N \rightarrow \infty$.

Proof: This is proven using the result stated in Lemma 4.1. The interested reader should consult [5] for exact details.

Assumption 4.1 The sequence $\left\{x_{N}^{*}\right\}_{N=1}^{\infty}$ converges as $N \rightarrow$ $\infty$.
Under Assumption 4.1, we have the following strong convergence for the sequence $x_{N}^{*}$.

Proposition 4.2 Let $x^{*}$ be the optimal solution for $(P)$ and $x_{N}^{*} \in X_{N}$ be the optimal solution for $\left(P_{N}\right)$. Under Assumption 4.1, $x_{N}^{*} \rightarrow x^{*}$ as $N \rightarrow \infty$.

Let $z_{N}^{*}$ be the optimal cost for $\left(P_{N}\right)$ and $\left(D_{N}\right)$. For primal feasible $x$ and dual feasible $\lambda$ we have

$$
-\frac{1}{2^{N}} \sum_{i=1}^{m} \sum_{i=1}^{2^{N}} \lambda_{i j} b_{i}\left(\eta_{j}\right) \leq z_{N}^{*} \leq\left\langle c_{N}, x\right\rangle
$$

The potential function for $\left(P_{N}\right)$ where $z \leq z_{N}^{*}$ is

$$
\phi_{N}(x, z)=\rho \ln \left(\left\langle c_{N}, x\right\rangle-z\right)-\frac{1}{2^{N}} \sum_{i=1}^{m} \sum_{i=1}^{2^{N}} \ln s_{i}\left(x, \eta_{j}\right)
$$

Analogous to (10), the Newton step $\pi_{N} \in X_{N}$ for $\left(P_{N}\right)$ is

$$
\pi_{N}(x, z)=\frac{Q_{N}(x)^{-1} \cdot \nabla \phi_{N}(x, z)}{\left\langle Q_{N}(x)^{-1} \cdot \nabla \phi_{N}(x, z), \nabla \phi_{N}(x, z)\right\rangle^{\frac{1}{2}}}
$$

where

$$
Q_{N}\left(x_{N}^{0}\right)=\frac{1}{2^{N}} \sum_{i=1}^{m} \sum_{j=1}^{2^{N}} \frac{a_{i}\left(\eta_{j}\right) \otimes a_{i}\left(\eta_{j}\right)}{s_{i}\left(\eta_{j}, x_{N}^{0}\right)^{2}}
$$

It can be shown that $\lambda_{i j}^{(N)}=f_{i}^{(N)}\left(x_{N}^{0}, z_{N}^{0}, \eta_{j}\right)$ where

$$
\begin{gathered}
f_{i}^{(N)}\left(x_{n}^{0}, z_{N}^{0}, \eta\right)=\left[\frac{\left\langle c_{N}, x_{N}^{0}\right\rangle-z_{N}^{0}}{\rho}\right]\left[s_{i}\left(\eta, x_{N}^{0}\right)^{-1}\right. \\
\left.\left\langle\frac{a_{i}(\eta)}{s_{i}\left(x_{N}^{0}, \eta\right)^{2}}, Q_{N}\left(x_{N}^{0}\right)^{-1} \cdot \nabla \phi_{N}\left(x_{N}^{0}, z_{N}^{0}\right)\right\rangle\right]
\end{gathered}
$$

is dual feasible for $\left(D_{N}\right)$. Given $z_{N}^{0} \leq z_{N}^{*}$, the lower bound update equation analogous to $(Z P)$ is

$$
\left(Z P_{N}\right)\left\{\begin{array}{c}
z_{N}^{1}=\arg \max z \\
f_{i}^{(N)}\left(x^{0}, z, \eta_{j}\right) \geq 0 ; i=1, \cdots, m ; \eta_{j} \in I_{N} \\
z \geq z_{N}^{0}
\end{array}\right.
$$

For $\left(P_{N}\right)$, we have the following algorithm:

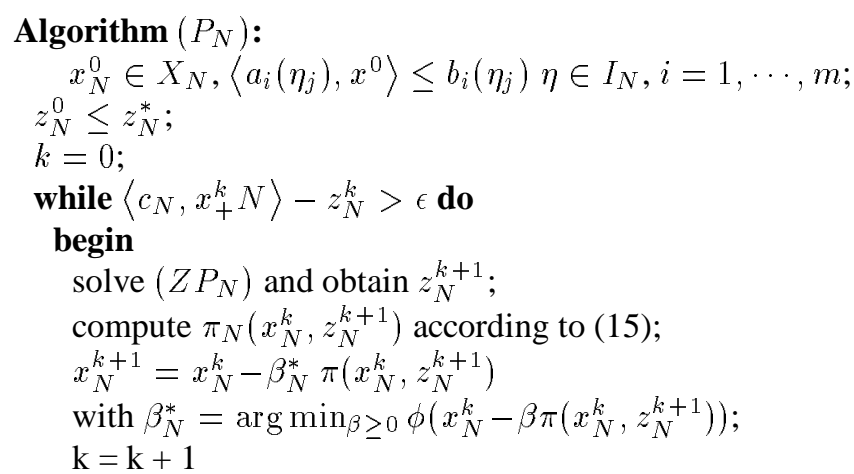




\section{end \\ end}

By simple modifications of the proofs in [9], it can be shown that the iterates $\left(x_{N}^{k}, z_{N}^{k}\right)$ produced by Algorithm $\left(P_{N}\right)$ have the following convergence results.

Proposition 4.3 If $x_{N}^{k}, k \in \mathbf{Z}^{+}$is the sequence of feasible solutions produced by Algorithm $\left(P_{N}\right)$ for the problem $\left(P_{N}\right)$, then $x_{N}^{k} \rightarrow x_{N}^{*}$ as $k \rightarrow \infty$.

In fact, we need a stronger statement about convergence of the iterates $x_{N}^{k}$ than is stated in Proposition 4.3, namely that $x_{N}^{k} \rightarrow x_{N}^{*}$ uniformly. We shall assume that this is true. A precise statement of this is as follows:

Assumption 4.2 For all $\epsilon>0$ there exists $N, \bar{q}$ such that $\left\|x_{q}^{k}-x_{q}^{*}\right\|<\epsilon$ for all $q>\bar{q}$ and $k>N$.

Let $x^{k} \in X$ be feasible for $(P)$ and $x_{N}^{k} \in X_{N}$ be feasible for $P_{N}$. We denote by $z_{N}^{k+1}$ the optimal solution of $\left(Z P_{N}\right)$ associated with $x_{N}^{k}$, and $z^{k+1}$ the optimal solution of $(Z P)$ with $x^{k}$. We have the following result.

The following assumption is required in some of the results that follow.

Assumption 4.3 Let $z^{*}$ be optimal for $(Z P)$ associated with $x \in X$. For all $\epsilon>0$, there exists $z \in \mathbf{R}$ such that $z^{*}>z>$ $\underline{z}^{*}-\epsilon$ and $f_{i}(x, z, \eta)>0$ for all $\eta \in[0,1]$.

Lemma 4.2 Let $x^{k}, x_{N}^{k}$ and $z_{N}^{k+1}$ be as above. Suppose that $x_{N}^{k} \rightarrow x^{k}$ as $N \rightarrow \infty$. If there exists $\bar{N} \in \mathbf{Z}^{+}$and $\bar{z}>-\infty$ such that $\bar{z}<z_{N}^{k+1}<\infty$ for each $N>\bar{N}$, then $(Z P)$ has an optimal solution $z^{k+1}$ and $z_{N}^{k+1} \rightarrow z^{k+1}$ as $N \rightarrow \infty$.

Proof: Refer to [5] for details

We consider now the convergence of the Newton step.

Lemma 4.3 Let $k \in \mathbf{Z}^{+}$be given. For every $N \in \mathbf{Z}^{+}$let $x_{N}^{k}$ be feasible for $\left(P_{N}\right), x^{k}$ be feasible for $(P)$ with $x_{N}^{k} \rightarrow x^{k}$ as $N \rightarrow \infty$. If $z_{N}^{k+1} \leq z_{N}^{*}$ and $z^{k+1} \leq z^{*}$ are as in Lemma 4.2 then $x_{N}^{k+1} \rightarrow x^{k+1}$ as $N \rightarrow \infty$.

Proof: We give a brief outline of the proof. For complete details, the reader should consult [5]. Let

$$
\begin{aligned}
\beta_{N}^{*} & =\arg \min _{\beta \geq 0} \phi_{N}\left(x_{N}^{k}-\beta \pi_{N}\left(x_{N}^{k}, z_{N}^{k+1}\right), z_{N}^{k+1}\right) \\
\beta^{*} & =\arg \min _{\beta \geq 0} \phi_{N}\left(x^{k}-\beta \pi\left(x^{k}, z^{k+1}\right), z^{k+1}\right)
\end{aligned}
$$

In [5] it is shown that $\pi_{N}\left(x_{N}^{k}, z_{N}^{k+1}\right) \rightarrow \pi\left(x^{k}, z^{k+1}\right)$ and $\beta_{N}^{*} \rightarrow \beta^{*}$ as $N \rightarrow \infty$. The result follows.

Lemma 4.4 Let $\left\{x_{N}^{k}\right\}_{k=0}^{\infty}$ and $\left\{x^{k}\right\}_{k=0}^{\infty}$ be iterates produced by $\left(P_{N}\right)$ and $(P)$ respectively. For every $k \in \mathbf{Z}^{+} x_{N}^{k} \rightarrow x^{k}$ as $N \rightarrow \infty$.
Proof: We prove this using induction.

This is true for $k=0$, for let $x^{0} \in X$ be feasible for $(P)$. Denote by $x_{N}^{0}$ the projection of $x^{0}$ onto $X_{N}$. Then $x_{N}^{0}$ is feasible for $\left(P_{N}\right)$ and $x_{N}^{0} \rightarrow x^{0}$ as $N \rightarrow \infty$. Let $z^{1} \leq z^{*}$ solve $(Z P)$ and $z_{N}^{1} \leq z_{N}^{*}$ solve $\left(Z P_{N}\right)$. Then by Lemma $4.2 z_{N}^{1} \rightarrow z^{1}$ as $N \rightarrow \infty$. Thus $\left\{\left(x_{N}^{0}, z_{N}^{1}\right)\right\}_{N=1}^{\infty}$ satisfies the conditions of Lemma 4.3 and $x_{N}^{1} \rightarrow x^{1}$ as $N \rightarrow \infty$.

Suppose there is $k \in \mathbf{Z}^{+}$such that $x_{N}^{k} \rightarrow x^{k}$ and $z_{N}^{k+1} \rightarrow$ $z^{k+1}$ as $N \rightarrow \infty$. Then the conditions of Lemma 4.2 are satisfied and hence, $x_{N}^{k+1} \rightarrow x^{k+1}$ as $N \rightarrow \infty$.

The result follows from induction.

Under Assumptions 2.1, 2.2, 2.3, 4.2 and 4.3, the sequence of interates $\left\{x^{k}\right\}_{k=1}^{\infty}$ produced by $\operatorname{Algorithm}(P)$ has the following weak convergence properties:

Theorem 4.1 If $\left\{x^{k}\right\}_{k=1}^{\infty}$ be the sequence of iterates produced by Algorithm $(P)$ and $x^{*}$ is an optimal solution of $(P)$ then $x^{k} \rightarrow x^{*}$ as $k \rightarrow \infty$.

Proof: Let $\left\{x_{N}^{k}\right\}_{k=1}^{\infty}$ be the sequence of iterates which result from applying Algorithm $\left(P_{N}\right)$ to $\left(P_{N}\right)$. Let $\epsilon>0$ and $z \in X$ be given. By Lemma $4.4, x_{p}^{k} \rightarrow x^{k}$ as $p \rightarrow \infty$. Since strong convergence implies weak convergence, there exists $N_{1} \in \mathbf{Z}^{+}$such that $\left\langle z, x^{k}-x_{p}^{k}\right\rangle\left\langle\frac{\epsilon}{3}\right.$ for all $p>$ $N_{1}$. By Proposition 4.3 and Assumption $4.2 x_{q}^{k} \rightarrow x_{q}^{*}$ uniformly so there exists $K \in \mathbf{Z}^{+}$and $N_{2} \in \mathbf{Z}^{+}$such that $\left\langle z, x_{q}^{k}-x_{q}^{*}\right\rangle<\frac{\epsilon}{3}$ for all $k>K$ and $q>N_{2}$. By Proposition 4.1, there exists $N_{3} \in \mathbf{Z}^{+}$such that $\left\langle z, x_{q}^{*}-x^{*}\right\rangle<\frac{\epsilon}{3}$ for all $q>N_{3}$. Thus, with $p, q>\max \left\{N_{1}, N_{2}, N_{3}\right\}$ and $k>K$, we have $\left|\left\langle z, x^{k}-x^{*}\right\rangle\right| \leq\left|\left\langle z, x^{k}-x_{q}^{k}\right\rangle\right|+$ $\left|\left\langle z, x_{q}^{k}-x_{q}^{*}\right\rangle\right|+\left|\left\langle z, x_{q}^{*}-x^{*}\right\rangle\right|<\epsilon$.

An immediate consequence of the definition of weak convergence is the following:

Corollary 4.1 Let $\left\{x^{k}\right\}_{k=0}^{\infty}$ and $x^{*}$ be as in Theorem 4.1. Then $\left\langle c, x^{k}\right\rangle \rightarrow\left\langle c, x^{*}\right\rangle$ as $k \rightarrow \infty$.

If in addition we assume that Assumption 4.1 holds, we have the following strong convergence result:

Theorem 4.2 Let $\left\{x^{k}\right\}_{k=0}^{\infty}$ and $x^{*}$ be as in Theorem 4.1. Then $x^{k} \rightarrow x^{*}$ as $k \rightarrow \infty$.

Proof: The proof is similar in spirit to that of Theorem 4.1, and requires in addition, the result stated in Proposition 4.2. The interested reader should consult [5] for details.

\section{Example}

As with all interior point methods, a crucial part of Algorithm is calculating the Newton step (9). We consider now a class of infinite-dimensional LP problems which are known as continuous linear programming (CLP) [7]. By placing additional differentiability constraints on the set of feasible solutions, this class of problems can be transformed into a constrained linear optimal control problem which falls under the framework $(P)$. We shall focus on calculating the Newton step. 
The $(C L P)$ problem is stated as follows:

$$
(C L P)\left\{\begin{array}{c}
\int_{0}^{1} c^{\prime}(t) x(t) d t \rightarrow \min \\
B(\eta) x(\eta)+\int_{0}^{1} K(\eta, t) x(t) d t \leq b(\eta) \\
x(\eta) \geq 0 ; \eta \in[0,1]
\end{array}\right.
$$

In addition, we shall assume that the $x \in L_{2}^{n}[0,1]$ and twice differentiable; that is there exists $y(t), u(t) \in L_{2}^{n}[0,1]$ such that

$$
\begin{aligned}
\dot{x}(t) & =y(t) \\
\dot{y}(t) & =u(t) \\
x(0), y(0) & =\gamma
\end{aligned}
$$

With this additional constraint, $(C L P)$ is a special case of the following constrained linear optimal control problem:

$$
(E)\left\{\begin{array}{c}
\int_{0}^{1} c^{\prime}(\tau) w(\tau) d \tau \rightarrow \min \\
\int_{0}^{1} a_{i}(\eta, \tau) w(\tau) d \tau \leq b_{i}(\eta) ; i=1, \cdots, m \\
w \in X
\end{array}\right.
$$

where

$$
\begin{gathered}
X=\left\{w \in L_{2}^{2 n}[0,1]:\right. \\
\dot{w}(t)=A(t) w(t)+B(t) u(t), w(0)=0\} \\
\dot{w}(t)=A(t) \bar{w}(t) ; \quad \bar{w}(0)=0
\end{gathered}
$$

Then

$$
\begin{aligned}
X^{\perp}=\left\{\dot{p}+A^{\prime} p: \dot{p} \in L_{2}^{2 n}[0,1],\right. \\
\\
p(1)=0, p \text { abs cts on }[0,1]\}
\end{aligned}
$$

For $(E)$, we have the potential function

$\phi(w, z)=\rho \ln \left[\int_{0}^{1} c^{\prime}(\tau) w(\tau) d \tau-z\right]-\int_{0}^{1} \ln s_{i}(w, \tau) d \tau$

The Newton step (9) which we denote by $\xi\left(w^{0}, z^{0}\right)$ is

$$
\xi\left(w^{0}, z^{0}\right)=-Q\left(w^{0}\right)^{-1} \cdot \nabla \phi\left(w^{0}, z^{0}\right)
$$

where

$$
Q\left(w^{0}\right)=\sum_{i=1}^{m} \int_{0}^{1} \frac{a_{i}(\eta, t) \cdot a_{i}^{\prime}(\eta, t)}{s_{i}\left(\eta, w^{0}\right)^{2}} d \eta
$$

When there is no risk of confusion, we shall denote $\xi\left(w^{0}, z^{0}, t\right)$ by $\xi(t)$. Substituting (21) into (20) gives

$$
\begin{aligned}
& -\nabla \phi\left(w^{0}, z^{0}, t\right)= \\
& \quad \sum_{i=1}^{m} \int_{0}^{1} \frac{a_{i}(\eta, t)}{s_{i}\left(\eta, w^{0}\right)^{2}}\left(\int_{0}^{1} a_{i}^{\prime}(\eta, \tau) \xi(\tau) d \tau\right) d \eta
\end{aligned}
$$

Now $\xi(t) \in X$ so

$$
\xi(t)=\int_{0}^{t} \Phi(t, \tau) B(t) r(\tau) d \tau
$$

for some $r(t) \in L_{2}^{m}[0,1]$ where $\Phi(t, \tau)$ is the state transition matrix associated with the state equation which defines $X$ in (18). Substituting (23) into (22) gives

$$
\begin{aligned}
& -\nabla \phi\left(x^{0}, z^{0}, t\right)= \\
& \sum_{i=1}^{m} \int_{0}^{1} \int_{0}^{1} \frac{a_{i}(\eta, t) a_{i}^{\prime}(\eta, \tau)}{s_{i}\left(\eta, w^{0}\right)^{2}} \int_{0}^{\tau} \Phi(\tau, \theta) B(\theta) r(\theta) d \theta d \tau d \eta
\end{aligned}
$$

Therefore, to calculate the Newton step, one needs to solve the integral equation (24) for $r(t)$ and (23) for the Newton step $\xi(t)$. In practise it may only be possible to solve (24) approximately - for example, partitioning $[0,1]$ and constraining $r(t)$ to be piecewise constant on each subinterval. However, the Newton step $\xi(t)$ obtained by solving (23) with this piecewise constant $r(t)$ will still satisfy the condition $\xi(t) \in X$.

\section{References}

[1] L. Faybusovich and J.B. Moore. A long-step pathfollowing algorithm for the convex quadratic programming problem in a Hilbert space. Proc. 34th IEEE Conference on Decision and Control.

[2] L. Faybusovich and J.B. Moore. Infinite dimensional quadratic optimization: interior-point methods and control applications. Journal of Applied Mathematics and Optimization (to appear).

[3] M.C. Ferris and A.B. Philpott. An interior point algorithm for semi-infinite programming. Mathematical Programming, no 43, 1989, pp 257 - 276.

[4] M.C. Ferris and A.B. Philpott. On affine scaling and semi-infinite programming. Mathematical Programming, no 56, 1992, pp $361-364$.

[5] A.E.B. Lim. A potential reduction method for semiinfinite linear programming on a Hilbert space. (In preparation).

[6] M.J.D. Powell. Karmarkar's algorithm: a view from nonlinear programming. Bulletin of the Institute of Mathematics and its Applications, no 26, 1990, pp $165-181$.

[7] M.C. Pullan. An algorithm for a class of continuous linear programs, SIAM J. Control Optim., 31, 1993, pp $1558-1577$.

[8] M.J. Todd. Interior-point algorithms for semi-infinite programming Mathematical Programming, no 65, 1994, pp $217-245$.

[9] Y. Ye. An $O\left(n^{3} L\right)$ potential reduction algorithm for linear programming. Mathematical Programming, no 50, 1991, pp $239-258$. 